\title{
Animación sociolaboral y educación social: perfil profesional en el municipio de
}

\section{Ourense}

\section{Social and occupational animation and social education: professional profile in Ourense municipality}

\author{
Laura Estévez Carballeira*, Ma Reyes Fernández González**. María D. Dapía Conde** \\ **Facultade Ciencias da Educación. Campus de Ourense. Universidade de Vigo.
}

\begin{abstract}
Resumen
El acceso al trabajo es uno de los instrumentos más importantes de integración social. Si la Educación Social se concibe como un instrumento de mejora de la conducta del ser humano en sus relaciones con los demás, pero también de la misma sociedad que crea los desajustes que hacen necesaria la intervención del educador y educadora social, no es de extrañar que la Animación Sociolaboral sea una de sus salidas laborales. Con el fin de conocer el perfil profesional de estos titulados y tituladas, que en el municipio de Ourense están realizando funciones de Animación Sociolaboral, se presenta esta investigación. Palabras clave: educación social, transición escuela-trabajo, inserción laboral.
\end{abstract}

\section{Abstract}

Access to work is one of the most important instruments of social integration. If Social Education is conceived as an instrument for improving the behavior of the human being in his relations with others, but also with the same society that creates the maladjustments that need the men and women community workers interventions, it won't surprising that the Social and occupational Animation is one of his professional outlets. This research is summitted in order to know the professional profile of these men and women graduates, who are making functions(roles) of Sociolaboral Animation in Ourense municipality.

Keywords: words: social education, school-work transition, labour insertion.

El hecho de tener un empleo o carecer del mismo influye notablemente en todas las dimensiones del ser humano, ya que, el trabajo cumple tres funciones fundamentales, que responden a tres necesidades básicas del individuo, y son: función económica, cubre las necesidades de subsistencia más básicas; función social, satisface la necesidad personal de colaboración y relación; y, función psicológica, cubre la necesidad de crear, de desarrollo y de autorrealización (Blanch, 1990; Salanova, Gracia y Peiró, 1996).

Pero, en este momento, a principios de la segunda década del siglo XXI, podemos decir que atravesamos un paro cíclico histórico. Después de la crisis económica de los años 20, la crisis de los 70 y la de los 90 en el siglo $\mathrm{XX}$, estamos viviendo la primera gran recesión económica del siglo XXI (Jiménez, 2013), lo que ha provocado un altísimo índice de paro y favorecido que el número de personas y/o familias en riesgo de exclusión social haya aumentado considerablemente.

Además, los efectos del cambio social o tecnológico han hecho obsoletos rápidamente muchos oficios y profesiones, creando problemas de adaptación ocupacional al existir un desajuste entre las necesidades siempre cambiantes del mercado y los perfiles profesionales de las personas demandantes de empleo (Iturbide y Serrano, 2004), por lo que se hace necesario tener actualizados los requisitos laborales.

$\mathrm{Y}$ es, en este contexto, donde se ubican las funciones a desenvolver por los educadores y las educadoras sociales dentro del ámbito de la Animación Sociolaboral (ASL).

La Animación Sociolaboral se configura como un "método de trabajo en el que se aúnan las prácticas, métodos y experiencias de la Animación Sociocultural y de la Orientación Laboral" (Maya y Caballero, 2001, p. 20); y se puede definir como una manera de intervención socioeducativa, que se concreta en la elaboración y desarrollo de un proyecto, implementado por profesionales, que trabajan para estimular la participación de los individuos en su propio desarrollo, concienciándolos de sus posibilidades de inserción, y así favorecer su integración sociolaboral (Fernández González y Dapía Conde, 2015).

En otras palabras, la ASL no es sólo una metodología que ayuda a través de acciones formativas de diversa temática -conocimientos profesionales, competencias transversales, técnicas de búsqueda de empleo- a la mejora de la empleabilidad de la población activa en situación de desempleo, sino que incorpora en su proyecto un conjunto de prácticas sociales que pretenden estimular la participación de las comunidades en el proceso de su propio desarrollo y en la dinámica global de la vida sociopolítica en la que están integradas.

Debido a esta última característica es por lo que habitualmente se consideran como usuarios o usuarias tipo de los proyectos de ASL a los colectivos en riesgo de exclusión social, por ser los que se encuentran en la posición más complicada ante los nuevos retos de las modernas sociedades: jóvenes, mujeres, personas 
paradas mayores de 40 años, inmigrantes, personas excluidas, etc. (Maya y Caballero, 2001, 36).

En lo que respecta al perfil profesional, es importante matizar que son muchas las figuras que se pueden incluir dentro de los y las profesionales de la Animación Sociolaboral -animador/a, orientador/a, técnico/a, intermediador/a y prospector/a-, pero todas ellas comparten la misma finalidad: optimizar la empleabilidad de las personas participantes en los diferentes proyectos que se estén llevando a cabo. "Su competencia general es informar, orientar, asesorar, motivar, programar formación, desarrollar competencias, realizar acciones de intermediación laboral y de prospección de empresas, y evaluar todo el proceso seguido para promover la inserción sociolaboral de las personas" (Fernández González y Dapía Conde, 2015).

Siguiendo a las autoras anteriormente citadas, en la práctica, las tareas que comparten todos estos/as profesionales cuando centran su atención en lo laboral son:

- Diagnóstico de la situación ocupacional de la persona mediante una entrevista personalizada.

- Determinación de las líneas de actuación que favorecen la inserción laboral de la persona.

- Puesta en práctica de las actuaciones de inserción laboral y de las de inserción social, cuando se requieran.

- Canalizan la información referente a recursos y servicios sociales de ayuda a la situación concreta de la persona.

- Valoran los resultados de la intervención, con aplicación de las técnicas de evaluación adecuadas.

Por lo tanto, estas funciones se concretan en el diseño, la implementación y la evaluación de Itinerarios Personalizados de Inserción (IPIs). A pesar de que estos itinerarios se aplican desde hace muchos años, no disponemos de una definición unificada sobre qué son, pero sí existe un consenso sobre sus características (Red de Inserción Sociolaboral de Castilla-La Mancha, 2007, p. 7):

- Se concretan en un proceso que integra las distintas etapas de las que constan.

- Su objetivo es la mejora de la situación sociolaboral de las personas.

- Se operativizan en un plan de trabajo personalizado.

- Están dirigidos a personas con voluntad para trabajar, que se encuentren con alguna dificultad añadida al desempleo, generalmente, la carencia de competencias personales $\mathrm{y} / \mathrm{o}$ profesionales o la pertenencia a un colectivo en riesgo de exclusión.

- Son realizados por equipos de trabajo multidisciplinares, porque su enfoque es mixto al atender lo psicosocial y lo económico.

- Son soportados por una organización que gestiona los recursos.

- Deben incorporar elementos para la coordinación de los recursos y servicios que intervienen en el proceso de inserción sociolaboral.

La concreción de todos estos elementos en una definición, nos permite conceptualizar los IPIs como un proceso que integra una serie de servicios y recursos, distinguibles pero asociados y coordinados entre sí, que se ofrecen a un mismo individuo de forma adaptada a sus características, con el fin de incidir sobre las diferentes dimensiones de las problemáticas que obstaculizan su acceso efectivo al mercado de trabajo y así mejorar su situación sociolaboral.

Además, esta metodología al ser considerada un proceso, se estructura en fases, que aunque pueden adoptar otra terminología a la aquí explicada, deben respetar la lógica siguiente de secuenciación (Red de Inserción Sociolaboral de Castilla-La Mancha, 2007, p. 13):

a) Acceso. El personal técnico realiza una entrevista para recoger información sobre la situación sociolaboral de la persona en búsqueda de empleo e identificar los costes de oportunidad.

b) Diagnóstico de la empleabilidad, con la finalidad de conocer más detalladamente la situación sociolaboral, identificar los signos que verifican la situación de vulnerabilidad y riesgo de exclusión, establecer las expectativas y necesidades percibidas y jerarquizar necesidades.

c) Plan de trabajo consensuado con la persona, ajustando sus expectativas para prevenir situaciones de frustración. Su estructura es la siguiente: identificación del/a usuario/a y del/a técnico/a, objetivos, resultados esperados, actividades a desarrollar por la persona usuaria y por el personal técnico, incentivos, derivaciones a otros servicios y recursos, y cronograma para el seguimiento y evaluación.

d) Derivación. Coordinación y complementariedad entre los diferentes recursos que ofrecen servicios relacionados con la inserción sociolaboral.

e) Seguimiento y evaluación para comprobar o verificar que los objetivos se cumplieron.

f) Intermediación. Acercamiento activo a las empresas para dar a conocer el perfil de cada candidatura y promover un marco de confianza para la contratación, porque los usuarios/as tienen dificultades en el acceso al empleo, pero no a la hora de realizar su trabajo.

\section{Objetivos}

El objetivo general sobre el que se asienta la presente investigación es describir el perfil profesional de los educadores y las educadoras sociales que llevan a cabo actividades de Animación Sociolaboral en el municipio de Ourense. Para conseguir este objetivo, es necesario:

- Identificar y describir las instituciones ubicadas en el municipio de Ourense que llevan a cabo programas/actividades de ASL.

- Determinar en cuáles de esas instituciones los y las profesionales que implementan esos programas/actividades poseen la titulación de Educación Social -diplomatura o grado-.

- Aproximarse a su desempeño profesional, analizando el perfil de las personas destinatarias, las funciones desarrolladas relacionadas con la inserción sociolaboral y el equipo de profesionales con los que trabaja. 
- Describir la formación y competencias que debería poseer un profesional de la inserción sociolaboral.

- Conocer las condiciones laborales en las que estos educadores y educadoras sociales desarrollan su trabajo: denominación del puesto, tipo de contrato formalizado, jornada, horario.

- Describir el acceso al puesto de trabajo: vías de búsqueda de empleo, requisitos previos, proceso de selección.

- Conocer la valoración que estos/as profesionales de la ASL realizan tanto de la formación recibida en su titulación universitaria, como de la complementaria realizada, en relación al puesto desempeñado.

\section{Participantes}

Para conocer las entidades sociales establecidas en el municipio de Ourense, iniciamos su búsqueda en el Registro Único de Entidades Prestadoras de Servicios Sociales, ubicado en la web de la Consellería de Política Social de la Xunta de Galicia (https://politicasocial.xunta.gal/XiacWeb/), resultando un total de 131 entidades y 85 centros.

Tras un primer filtrado atendiendo a su denominación, personas destinatarias, conocimiento de sus actividades, así como mediante la consulta de las páginas webs de aquellas entidades y centros en las que podría haber dudas respecto a sí incluían a no entre sus acciones programas o actividades relacionadas con la inserción sociolaboral, se llegó a un listado de 23 entidades.

Finalmente, resultaron ser un total de 11 entidades las que cumplían nuestros requisitos: entidades que realizan acciones de ASL implementadas por un educador o una educadora social en el momento de realizar la investigación, enero de 2016, y que están ubicadas en el municipio de Ourense. Dos de ellas no participaron en nuestra investigación por imposibilidad de hacer la entrevista. Por tanto, son 9 las entidades que participan y 10 las personas entrevistadas, puesto que en una de las asociaciones dos trabajadores/as decidieron hacerla de manera conjunta. Si bien a efectos de esta investigación vamos a contar sólo como si esta entrevista fuera realizada por una persona, ya que uno/a de estos dos trabajadores/as realizaba sus funciones en el programa de ocio de la entidad, sin llevar a cabo ninguna función relacionada con la inserción sociolaboral.

Por lo tanto, las entidades que participaron en nuestra investigación fueron:

- Asociación Down Ourense (ADO)

- AIXIÑA

- Asociación Para la Prevención Social (APES)

- Cáritas

- Cruz Roja

- R.R. Adoratrices Esclavas Santísimo Sacramento y Caridad de Ourense (ÉRGUETE MULLER)

- Instituto Galego de Xestión para o Terceiro Sector (IGAXES)

- RENACER

- Fundación Juan Soñador (Programa TERANGA)

Además, se valoró la inclusión de alguno de los centros que el propio ayuntamiento de Ourense posee y que pueden trabajar para favorecer la inserción sociolaboral de sus usuarios y usuarias, como por ejemplo el Centro de Información á Muller (CIM) o las Unidades de Intervención Social (UNIS). Estas entidades quedaron descartadas, bien por no tener la figura del Educador o Educadora Social o bien por no llevar a cabo acciones de Animación Sociolaboral, puesto que sólo se encargaban de derivar a las personas a otros servicios centrados en esta problemática.

\section{Instrumento}

El instrumento elegido para la recogida de datos fue la entrevista, por ser la técnica que mejor se adaptaba a nuestra investigación de corte cualitativo. Es una entrevista focalizada al centrar las preguntas en el tema de estudio, perfil profesional de los educadores y educadoras sociales que realizan acciones relacionadas con la Animación Sociolaboral en el municipio de Ourense; semiestructurada, puesto que la persona que va a realizar la entrevista tiene plena libertad para introducir preguntas adicionales con el fin de aclarar conceptos $u$ obtener mayor información sobre un tema en particular; $\mathrm{y}$, es en profundidad, puesto que sus preguntas son abiertas.

Basándonos en las entrevistas utilizadas por la profesora Dapía (2016) en sus investigaciones sobre los perfiles profesionales de Educación Social, y teniendo en cuenta la adecuación entre los contenidos de las preguntas y los objetivos de la investigación, diseñamos una entrevista que, además de contar con unos datos introductorios que pretendían identificar la entrevista y recoger algunos datos personales considerados básicos (sexo, titulación y datos de contacto) de las personas entrevistadas, presentaba 25 preguntas abiertas agrupadas en cuatro bloques:

- Desempeño profesional: 13 ítems.

- Condiciones laborales: 4 ítems.

- Acceso al puesto de trabajo: 6 ítems.

- Valoración formación: 2 ítems.

\section{Resultados}

A continuación se presentan los resultados de nuestra investigación, tras la categorización de la información obtenida en las entrevistas realizadas según la estructura de las mismas y atendiendo a los objetivos anteriormente planteados.

La mayoría de las personas entrevistadas fueron mujeres, un $77.77 \%$ frente a un $22.22 \%$ de hombres, hecho coherente con el carácter feminizado que presentan los estudios universitarios de Educación Social.

Podemos confirmar que un $52.38 \%$ de las entidades que trabajan a favor de la inserción sociolaboral de colectivos en riesgo de exclusión social en el municipio de Ourense han contratado personas tituladas en Educación Social para realizar actividades y/o programas de ASL. De ellas, 6 eran diplomados o diplomadas, 2 graduados o graduadas y una acababa de finalizar el curso puente para pasar de la diplomatura al grado.

Respecto a su desempeño profesional, podemos afirmar que en un $77.77 \%$ de las entidades entrevistadas trabajan con Itinerarios Personalizados de Inserción 
(IPIs) dirigidos a colectivos diversos, principalmente personas con discapacidad, destacando sobre el resto, seguidas de personas en situación de vulnerabilidad y exclusión social, menores, mujeres, personas privadas de libertad e inmigrantes, emigrantes retornados/as y familias de procedencia extranjera.

La mayoría de los y las profesionales realizan todas sus fases y otras, realizan solo una parte del IPI. El 22.23\% restante trabaja la inserción sociolaboral de manera transversal, destinando una pequeña parte de su jornada a la realización de acciones que pretenden mejorar la empleabilidad. El trabajo de estos/as profesionales suele ser integral, destinando una gran parte de su tiempo a la formación y a realizar acompañamientos. Consecuentemente, las funciones principales desarrolladas en el puesto de trabajo están todas relacionadas con el diseño e implementación de las distintas fases que componen los IPIs.

Para indagar más en el perfil de estos/as profesionales, nos interesó conocer las competencias/habilidades que consideraban necesarias para desarrollar sus funciones. La mayoría de los educadores y educadoras coincidieron en sus respuestas. Las más destacadas fueron aquellas que se relacionan con el trato a las personas, la comunicación y las habilidades sociales, en concreto la empatía y el trabajo en equipo.

Si hablamos de las actividades podemos afirmar que 6 de las entidades participantes cuentan con programas de empleo, una cuenta con un Centro Especial de Empleo y las restantes trabajan el tema de empleo de manera transversal dentro de sus programas.

Consultamos el equipo de trabajo y su constitución, atendiendo a las profesiones y el número de educadores y educadoras sociales con las que cuenta. La mayoría sostiene que es un equipo multidisciplinar. Por ello, encontramos diferentes perfiles profesionales, pertenecientes en la mayoría de los casos al ámbito jurídico-social:

- otro/a educador/a social, un 70\%,

- el/la trabajador/a social, profesional que en muchos casos forma gran parte de los equipos en un $60 \%$,

- el/la integrador/a social y el/la psicólogo/a, con una presencia a partes iguales, $\mathrm{y}$,

- otras titulaciones: Administración y Dirección de Empresas, Derecho, Logopedia, Fisioterapia, Terapia Ocupacional, Neuropsicología. Aclarar que estas 5 últimas profesiones son las que aparecen en las entidades que trabajan con personas con algún tipo de discapacidad.

Nos interesaba estar al tanto de si había otros compañeros o compañeras desempeñando funciones propias de la Educación Social sin poseer la titulación. La mayoría de las personas entrevistadas nos comentan que al existir representación de educadores/as sociales en su plantilla, no existe usurpación de funciones en su equipo, si bien las funciones se pueden solapar porque las competencias profesionales del educador o la educadora social siguen sin estar definidas.

Para profundizar en el perfil profesional de las personas que trabajan a favor de la inserción sociolaboral de los colectivos en riesgo de exclusión social, decidimos atender a sus condiciones laborales, comenzando con el análisis de la denominación de su puesto de trabajo. Un total de 6 de las 9 personas entrevistadas están contratadas como educadores/as sociales. Las otras denominaciones son técnico/a de inserción, encargado/a de servicios y educador/a. Una de ellas nos aclara que en su entidad, la denominación como educador/a social no existe, existe la de educador/a.

En cuanto al tipo de contrato, la mayoría goza de estabilidad, siendo sólo 2 las personas que están contratadas temporalmente. La jornada laboral, excepto una persona que trabaja a media jornada, es mayoritariamente a tiempo completo. Respecto al horario, 5 personas indican que es continuo frente a 4 que dicen tenerlo partido. Ninguno de los/as entrevistados/as trabaja los fines de semana y/o festivos. Apuntar que la gran mayoría de los y las profesionales reconocen que su horario no es fijo, sino que es flexible. Nos sorprendió gratamente la posibilidad del teletrabajo en un caso.

Para acceder al puesto de trabajo, a 7 de las personas entrevistadas se les requirió ser tituladas en Educación Social. A dos de ellas, además se les valoraba tener una formación complementaria. En cuanto a la experiencia, fue requisito imprescindible para 3 de los educadores y educadoras sociales entrevistados/as, si bien otros/as titulados/as no recuerdan si se les requirió porque estuvieron anteriormente en prácticas o realizando un voluntariado.

La vía de acceso al puesto de trabajo más empleada fue a través del Servicio Estatal Público de Empleo (SEPE, antiguo INEM), 5 personas, de las que una tuvo que usar obligatoriamente esa ente para poder formalizar un contrato a través de los Programas de Cooperación de la Xunta de Galicia. Otras personas entraron gracias a la autocandidatura, concretamente 2 . Y el resto, otras 2 personas, por otras vías: voluntariado y practicum de Educación Social. El proceso de selección consistió mayoritariamente en la realización de una entrevista, 7 personas. Para finalizar, les preguntábamos por la motivación para trabajar en temas relacionados con la inserción sociolaboral. La gran mayoría responde que fueron las circunstancias las que le llevaron a trabajar en este tema.

Pasamos ahora a analizar el apartado que se corresponde con la valoración de la formación. Encontramos aquí una diferencia en las respuestas referidas a si la formación recibida en la universidad les ha servido para desempeñar su actual trabajo. Son 3 las personas que sostienen que el paso por Educación Social no les ha ayudado a desempeñar su empleo. Son diferentes motivos los que salen a la luz tales como el carácter como optativas que tienen algunas materias y la falta de especialización. En cambio, son 6 las personas que afirman que la formación recibida en la carrera les ha servido para desarrollar su empleo y la consideran fundamental. A estas personas, les cuesta determinar cuáles son las materias que más han contribuido. Aun así, las que destacan son "Programas de Animación Sociocultural" y "Educación y Animación Sociolaboral". A la pregunta, ¿Has hecho o vas a hacer algún curso de especialización en el tema de la inserción sociolaboral?, $7 \mathrm{de}$ los/as entrevistados/as responde que sí ha hecho un curso de especialización, algunos lo han recibido por 
medio de la entidad para la que trabajan. Asimismo, 2 personas tienen intención de hacer un máster relacionado con el tema.

\section{Conclusiones}

Podemos afirmar que son muchas las entidades que cuentan con la figura del Educador o Educadora Social para trabajar la inserción sociolaboral.

Con respecto a los y las educadores y educadoras sociales, el perfil que encontramos en el municipio de Ourense es de mujer, diplomada en Educación Social, con formación complementaria a su titulación universitaria, y que desempeña su labor en entidades sin ánimo de lucro.

A la hora de trabajar en una entidad que tiene como finalidad la integración de las personas, cabe la posibilidad de que los/as profesionales de la Educación Social trabajen temas de inserción laboral. Las entidades que trabajan a favor de la inserción en el municipio de Ourense no descuidan la parte laboral, sino que la incluyen bien con programas o bien la trabajan de manera transversal.

Las funciones que desempeñan estos/as profesionales son las que se corresponden con las partes de un IPI, acompañamientos y gestión. Para desempeñarlas deben ser competentes y dominar las habilidades sociales y ser conscientes de con quien están desarrollando su intervención socioeducativa.

Los educadores y las educadoras sociales trabajan en equipo con distintos profesionales. Su constitución depende de la población objeto de las intervenciones. Aun así, la mayoría trabaja mano a mano con otro/a educador/a social y el/la trabajador/a social. A pesar de que en este equipo de trabajo están presentes más de un educador o educadora social, el hecho de que realicen variadas funciones, nos lleva a afirmar que siguen estando desdibujadas para los agentes empleadores y para la sociedad las funciones que debe realizar la Educación Social.

Podemos concluir que para trabajar en inserción laboral es necesario conocer el funcionamiento del mercado laboral, saber orientar y formar a la persona en cuanto a esas demandas. A su vez, el/la profesional tiene que estar en continua formación, dominar las habilidades sociales, trabajar en equipo y gestionar bien el tiempo.

En cuanto a las condiciones laborales en las que desempeñan su labor, podemos afirmar que estos/as profesionales trabajan como educadores/as sociales, con un contrato indefinido, empleados/as a tiempo completo. En cuanto al horario, no observamos diferencias relevantes entre continuo y partido, pero sí podemos afirmar que su horario es flexible y se adapta a las necesidades, incluso abriéndose paso la opción del teletrabajo.

En cuanto al acceso al puesto de trabajo, podemos decir que el requisito formativo imprescindible era la titulación universitaria en Educación Social. La experiencia no era imprescindible en todos los puestos de trabajo. La vía de acceso, en la gran mayoría de los casos, fue el SEPE y el proceso de selección más realizado la entrevista.
Analizamos la relación entre empleo y cursos de especialización, pudiendo decir que también está presente, y que su temática versa principalmente sobre la orientación laboral, el mercado laboral en Galicia o los certificados de profesionalidad:

Estos/as profesionales trabajan en temas relacionados con la inserción sociolaboral dependiendo de su momento de búsqueda de empleo y no tanto de su intención o vocación.

Con respecto a la valoración de la formación recibida en la carrera, podemos sostener que ésta es positiva moderadamente, ya que sólo señalan dos materias, "Programas de Animación Sociocultural" y "Educación y Animación Sociolaboral", por lo que todos/as consideran de vital importancia la constante actualización de sus conocimientos. La gran mayoría ha realizado cursos de especialización en el tema que nos compete.

\section{Referencias}

Blanch, J. M. (1990). Del viejo al nuevo paro. Un análisis psicológico y social. Barcelona: PPU.

Dapía, M. D. (2016). Investigación de los perfiles profesionales de educación social. El TFG, una oportunidad para la investigación aplicada. Educación Social. Revista de Intervención Socioeducativa, 62, 89-103.

Fernández González, M.R. y Dapía Conde, M.D. (2015). La Animación Sociolaboral, un ámbito emergente de la Educación Social. Sensos Revista Multimédia de Investigacão em Educação, 1,43-58.

Itúrbide, P. y Serrano, U. (2004). Manual de orientación laboral para personas con discapacidad. Loiu (Bizkais): Autor. Recuperado de http://sid.usal.es/idocs/F8/FDO10458/manualorient acion.pdf

Jiménez, M.J. (2013). Guía metodológica de la Inserción Laboral de las personas en situación de vulnerabilidad. Madrid: EAPN España.

Maya, P. y Caballero, J. J. (2001). El animador sociolaboral. Madrid: CCS.

Red de Inserción Sociolaboral de Castilla-La Mancha (2007). Guía para la aplicación del itinerario integrado y personalizado de inserción sociolaboral. http://empleoyformacion.jccm.es/fileadmin/user_up load/Fondo_Social_Europeo/Equal/GuiaItinerarios CLM08.pdf

Salanova, M., Gracia, F.J. y Peiró, J.M. (1996). Significado del trabajo y valores laborales. En J.M. Peiró y F. Prieto (eds.). Tratado de Psicología del Trabajo. Vol. II: Aspectos psicosociales del trabajo (pp. 35-64). Madrid: Síntesis. 